\title{
Inhibition of photodynamic therapy induced-immunosuppression with aminolevulinic acid leads to enhanced outcomes of tumors and pre-cancerous lesions (Review)
}

\author{
SHARLO BAYLESS ${ }^{1}$, JEFFREY B. TRAVERS ${ }^{1-3}$, RAVI P. SAHU ${ }^{1}$ and CRAIG A. ROHAN ${ }^{1,2}$ \\ Departments of ${ }^{1}$ Pharmacology and Toxicology, and ${ }^{2}$ Dermatology, Boonshoft School of Medicine, Wright State University, \\ Dayton, OH 45435; ${ }^{3}$ Deparment of Dermatology, Dayton Veterans Administration Medical Center, Dayton, OH 45428, USA
}

Received April 23, 2021; Accepted June 18, 2021

DOI: $10.3892 / 01.2021 .12925$

\begin{abstract}
Photodynamic therapy (PDT) is a treatment option for tumors and pre-cancerous lesions, but it has immunosuppressive side effects that limit its effectiveness. Recent studies suggest that PDT-mediated immunosuppression occurs through a cyclooxygenase type 2 (COX-2) mediated pathway that leads to increases in regulatory $\mathrm{T}$ cells (Tregs) and myeloid-derived suppressor cells (MDSCs), which act as negative regulators of immune responses. Given this pathway, there are three main methods to block immunosuppression: i) Inhibiting the proliferation of Tregs, which can be achieved with the administration of cyclophosphamide or inhibitors of indoleamine 2,3-dioxygenase 1, an activator of Tregs; ii) inhibiting MDSCs by reducing hypoxia around the tumor to create an unfavorable environment or administering all-trans-retinoic acid, which converts MDSCs to a non-immunosuppressive state; and iii) inhibiting COX-2 through selective or non-selective
\end{abstract}

Correspondence to: Dr Craig A. Rohan, Department of Pharmacology and Toxicology, Boonshoft School of Medicine, Wright State University, 3640 Colonel Glenn Boulevard, Dayton, OH 45435, USA

E-mail: craig.rohan@wright.edu

Abbreviations: PDT, photodynamic therapy; ROS, reactive oxygen species; ALA, 5-aminolevulinic acid; MAL, methyl aminolevulinate; PpIX, protoporphyrin IX; 5-FU, 5-fluorouracil; DNFB, dinitrofluorobenzene; MDSCs, myeloid-derived suppressor cells; Tregs, regulatory $\mathrm{T}$ cells; HSV, herpes simplex virus; TGF- $\beta$, transforming growth factor $\beta$; IL-10, interleukin-10; PAF, platelet-activating factor; COX-2, cyclooxygenase-2; PGE2, prostaglandin E2; IFN $\gamma$, interferon $\gamma$; IDO, indoleamine 2,3-dioxygenase; ATRA, all trans retinoic acid; ATP, adenosine triphosphate; PM-IR780-Met, platelet membranes as nano-carriers to co-encapsulate metformin and IR780; NSAID, nonsteroidal anti-inflammatory drugs; COX-1, cyclooxygenase-1

Key words: photodynamic therapy, immunosuppression, precancerous lesions, squamous cell carcinoma, actinic keratosis, pharmacologic therapy
COX-inhibitors. In the present review article, strategies that have shown increased efficacy of PDT in treating tumors and pre-cancerous lesions by blocking the immunosuppressive side effects are outlined and discussed.

\author{
Contents \\ 1. Introduction \\ 2. Overview of PDT-induced immunosuppression \\ 3. Inhibition of Tregs \\ 4. Inhibition of MDSCs \\ 5. Inhibition of COX-2 \\ 6. Conclusions
}

\section{Introduction}

Photodynamic therapy (PDT) is a form of treatment for tumors and pre-cancerous lesions that works by inducing cell death. The treatment involves a photosensitizing agent which is topically applied or injected into the blood stream $(1,2)$. It is absorbed by cells and after a pre-determined amount of time, the site is exposed to a light source such as a laser or light-emitting diode (1). When light is applied at a specific wavelength to the targeted area, the photosensitizing agent undergoes a reaction that forms reactive oxygen species (ROS) and kills the targeted cells $(1,2)$ and/or induces vascular damage (3). To the host, PDT is usually minimally invasive and minimally toxic as the photosensitizers do not tend to accumulate in cell nuclei $(2,4)$.

Photosensitizers are divided into families based on chemical structure including porphyrins, chlorins, and dyes (5). They are also grouped into first, second, and third generation photosensitizers (6). The first generation photosensitizer most commonly used is Photofrin, a mixture of porphyrin dimers and oligomers $(6,7)$. First generation photosensitizers are used less frequently today due to side effects such as skin sensitivity and their weak absorption at $630 \mathrm{~nm}(8,9)$. Two examples of second generation photosensitizers commonly employed in dermatology practice are aminolevulinic acid (ALA) and the methyl ester form, methyl aminolevulinate (MAL) (10). Both 
can be topically applied and have some selectivity for precancerous and cancerous cells, although MAL is more lipophilic and absorbed by deeper skin tissues than ALA (10). These agents are converted into the highly reactive protoporphyrin IX (PpIX) (11). Notably, rapidly proliferating cells, such as cancer cells, convert more ALA to PpIX in their mitochondria than their non-transformed counterparts in the epidermis. When neoplastic cells are exposed to light at various wavelengths (classically between 570 and $670 \mathrm{~nm}$ ) (10), ROS are created which then damage and destroy target cells. In clinical practice, both red (most commonly $630 \mathrm{~nm}$ ) and blue (410-420 nm) wavelength light sources are utilized. Moreover, 'daylight PDT' is also employed, using natural sunlight to activate these photosensitizing agents (10-12). Finally, third generation photosensitizers are antibody-directed and were developed to have a strong affinity for tumor cells, causing less damage to the surrounding tissues $(5,6)$

The clinical uses of PDT span from multiple neoplastic indications, skin disorders and ocular conditions. Considering the two-dose nature of the treatment, topical PDT is frequently favored over other field therapy options, even if reportedly less effective than other field therapy options such as 5-fluorouracil (5-FU) (13). It has been utilized for other dermatologic conditions, including psoriasis, basal cell carcinoma, verruca, and extramammary Paget's disease (14). Systemic PDT is also used to prevent severe vision loss in wet macular degeneration by targeting the vasculature that gives rise to the condition (15). Additionally, PDT has been used in patients with Barrett's esophagus, as well as cancers of the mouth and lungs (16).

However, there are multiple reports of side effects of PDT, including redness, pain and photosensitivity. Relevant to this review, PDT exerts immunomodulatory effects that could limit its effectiveness (17-23). This review paper highlights our current understanding of the immunosuppressive effects of PDT with ALA and will specifically focus on pharmacologic strategies to mitigate this unwanted effect, which has not specifically been reviewed in the current literature. The aim is to increase understanding of this process, which will be especially helpful in improving the effectiveness of topical PDT in treating tumors or pre-cancerous lesions.

\section{Overview of PDT-induced immunosuppression}

Despite the numerous indications that PDT is effectively used, there is strong evidence to suggest that PDT exerts local immunosuppressive effects (17-23). Those effects impact the overall success of the modality. Efforts to mitigate the resulting immunosuppressive effects could help improve efficacy or expand indications for PDT. For example, topical PDT has been associated with reactivation of orolabial herpes simplex virus (HSV) infections (24). Moreover, there is evidence from the literature, albeit anecdotal, that more aggressive melanomas and non-melanoma skin cancers can arise in PDT-treated skin $(25,26)$. However, this is a controversial point due to confounding variables such as the fact that skin treated with PDT was more likely to develop skin cancer. Notably, more solid evidence has been provided by preclinical studies. PDT was initially discovered to be immunosuppressive in mice using dinitrofluorobenzene (DNFB), a sensitizing agent that induces a contact hypersensitivity response. In this study, mice treated with PDT showed a significant decrease in their cell-mediated immune response to DNFB applied to the PDT-treated area (20).

When PDT is applied to an area, there is a rapid invasion of neutrophils, mast cells, monocytes, and macrophages to destroy abnormal tumor cells (27), demonstrating a robust anti-tumor immune response in the host. However, in addition to its immuno-stimulatory effects, PDT causes an increase in regulatory T cells (Tregs) and myeloid-derived suppressor cells (MDSCs) (28), which serve as counterregulatory mechanisms to impede the effector responses of the immune system (29). MDSCs secrete the immunosuppressive cytokines such as transforming growth factor-beta (TGF- $\beta$ ) and interleukin 10 (IL-10), which allows macrophages that migrate into a tumor to express inhibitor molecules (30) and converts Tregs to an active form, inhibiting effector T-cell proliferation (31). Tregs also suppress effector T cells directly, produce IL-10, and enhance MDSCs and regulate their differentiation through TGF- $\beta$ signaling (31). Hence, MDSCs and Tregs effectively 'cross-talk' through the B7-H1 pathway which directly suppresses T-cell proliferation to form an immunosuppressive microenvironment within tumors $(29,32)$ and thus favors tumor growth.

The exact mechanisms by which PDT exerts immunomodulatory effects is at present unclear. However, through its ability to generate ROS, PDT has been shown in cell lines and pre-clinical studies to be a potent generator of the lipid mediator, Platelet-activating factor (1-alkyl-2-acetyl-glycerophosphocholine, PAF) (33). Of note, exogenous PAF is known to induce systemic immunosuppression via its ability to generate Tregs (33). PAF upregulates IL-10, which inhibits the host immune response through the mechanism discussed earlier, but also leads to an increase in Tregs through a COX-2-mediated process $(34,35)$. COX-2 generates prostaglandins such as PGE2 which promote blood vessel formation, allowing increased angiogenesis resulting in enhanced growth/proliferation of experimental tumor types, including melanoma $(36,37)$. The activation of COX-2 is also connected to the induction and expansion of MDSCs (38) and increasing the levels of immunosuppressive Tregs (35), this mechanism was shown to exhibit its effects downstream of PAF in experimental models (34), with the overall pathway being $\mathrm{PAF} \rightarrow \mathrm{COX}-2 \rightarrow$ Tregs. Though demonstrated in mice, it has not yet been verified whether or not this pathway exists in humans.

Although PDT is an effective option in treating cancerous and precancerous cells, relapses can occur following treatment (39), likely due to the immunosuppressive side effects. This review is focused on studies, summarized in Table I, that have shown how blocking the immunosuppression can result in enhanced efficacy of PDT in decreasing tumors, pre-cancerous cells, and morbidity for patients. The relationship between MDSCs, Tregs, and COX-2 offers three targets for inhibiting PDT-induced immunosuppression, and illustrated in Fig. 1. These are the potentially targetable methods that will be explored in this review.

\section{Inhibition of Tregs}

Tregs are among the suppressive immunophenotypes which have been implicated in mediating immunosuppression or 


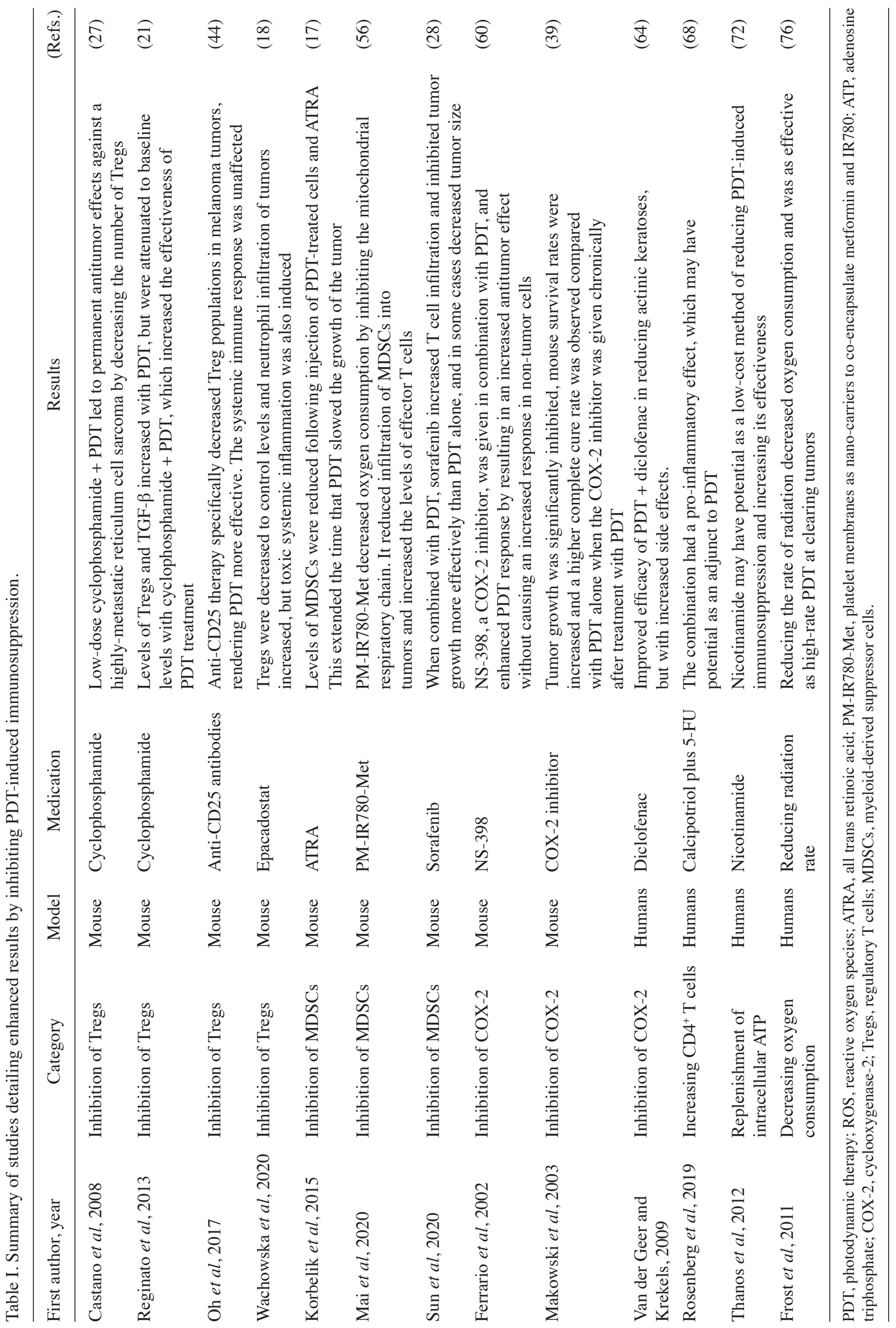




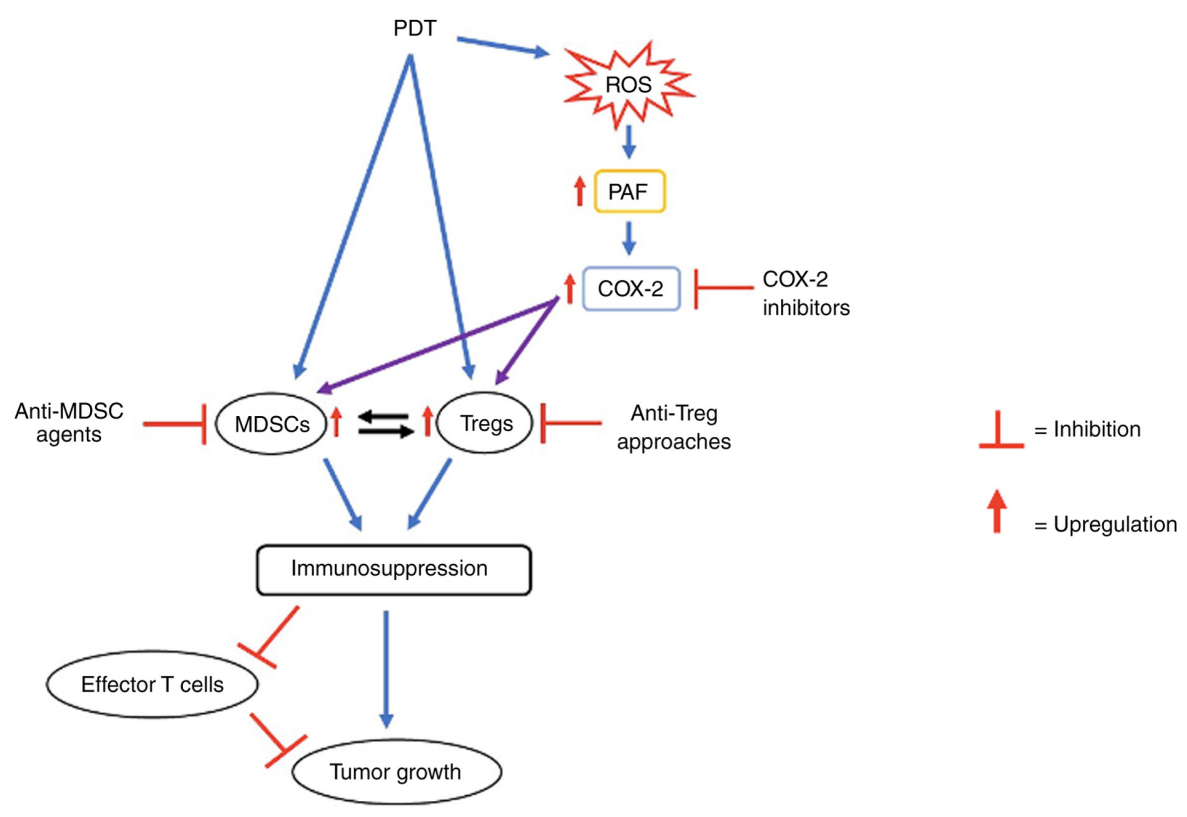

Figure 1. Schematic representation of the mechanisms involved in reducing the therapeutic efficacy of PDT. In this model, PDT, due to its prooxidant effects, generates ROS. As a consequence of this, PAF lipids are produced. The immunosuppressive cell types, Tregs and MDSCs, are upregulated via the downstream COX-2 pathway. This results in the immunosuppression and inhibition of effector T cells, leading to the reduced antitumor effect of PDT. Possible approaches to circumvent this PDT-induced immunosuppression and increase its efficacy include COX-2 inhibitors, anti-Treg approaches and anti-MDSC agents. PDT, photodynamic therapy; ROS, reactive oxygen species; PAF, platelet-activating factor; COX-2, cyclooxygenase-2; Tregs, regulatory T cells; MDSCs, myeloid-derived suppressor cells.

immune escape mechanisms (40). Multiple studies have demonstrated that Tregs proliferate at a larger extent in the tumor microenvironment, implicated in tumor progression and metastasis, as well as counterbalancing the anti-tumor immune responses of cancer therapies against malignancies including skin cancer $(41,42)$. To that end, Treg reprogramming has been explored as a critical approach to circumvent immunosuppressive mechanisms to control tumor growth and enhance the efficacy of therapeutic regimens $(42,43)$. As stated earlier, PDT activates the host's innate and adaptive immune systems, leading to a migration of inflammatory cells such as neutrophils and dendritic cells into the target area. However, a simultaneous immunosuppressive effect takes place in the tumor microenvironment, allowing it to evade the immune response. The first method of blocking this is to target the immunosuppressive Tregs.

One method is the administration of cyclophosphamide with PDT, the efficacy of which has been shown in multiple preclinical studies $(17,21,27)$. In a mouse model of a highly-metastatic reticulum cell sarcoma, PDT plus cyclophosphamide administered at a low dose caused a decrease in Tregs and increased the immune system's response to tumor growth $(21,27)$. PDT alone caused an increase in survival and tumor regression among mice, but no permanent cures. Cyclophosphamide alone also provided a survival advantage and reduced Tregs but led to no permanent cures. However, when PDT and cyclophosphamide were given together, the permanent cure rate was $70 \%$ (27). This synergistic effect was attributed to cyclophosphamide's ability to decrease the Treg population and prevent the immunosuppression induced by PDT (27).

In another study testing the effects of cyclophosphamide plus PDT, mice with colon carcinoma CT26 tumors were treated with either PDT alone or in combination with cyclophosphamide. The levels of Tregs were measured throughout treatment. Moreover, because TGF- $\beta$ is an immunosuppressive cytokine that both promotes the development of Tregs and allows Tregs to regulate MDSCs (31), levels of TGF- $\beta$ were also measured. The study showed that PDT alone leads to an increase in the Treg population, but that this effect is negated by the administration of cyclophosphamide before PDT, which brings down the Tregs to a level comparable to that of control mice (mice that were not inoculated with cancer) (21). Additionally, mice treated with PDT alone had an elevation in TGF- $\beta$, while mice treated with PDT and cyclophosphamide showed a significant decrease in TGF- $\beta$ levels that was similar to control mice (21). Furthermore, untreated mice survived a median of 25 days after tumor inoculation, while the median survival for mice treated with PDT alone was 29 days (21). However, 9 out of 10 mice treated with both cyclophosphamide and PDT displayed tumor regression, and all 9 of those mice survived over 90 days (21).

Tregs were also selectively depleted in the tumor microenvironment of mice in a study by Oh et al (44). This was achieved by injecting anti-CD25 antibodies that were conjugated to a photosensitizer, which induces apoptosis in Tregs (44). The purpose of this study was to find a method that decreased tumor-associated Treg populations without inducing severe autoimmune or hyper-immune systemic responses. Overall, tumor growth was inhibited by PDT plus the CD25-targeted therapy (44). The local tumor-associated Treg population was depleted without systemic side effects and the combination caused significant anti-tumor immunity at the site of the melanoma (44). The mice did not exhibit significant hyper-immune responses and continued to have an adaptive immune response against the influenza virus, demonstrating that the systemic immune response was not significantly affected (44). 
Another method to reduce the number of Tregs is to inhibit indoleamine 2,3-dioxygenase 1 (IDO). IDO is a heme-containing enzyme located in multiple tissues of the body that is expressed during inflammatory diseases and tumorigenesis (45). IDO is elevated after PDT and activates Tregs, preventing their conversion to effector T cells (46). Therefore, inhibiting IDO decreases Tregs and activates IL-6, which induces an acute inflammatory response (18). One study inoculated carcinoma tumor cells in murine models and showed that targeting IDO with inhibitors such epacadostat decreases Treg numbers to control levels and causes neutrophil infiltration of tumors, but also induces severe systemic inflammation at high doses of epacadostat through an IL-6 mechanism (18). The toxic reaction can be prevented with anti-IL-6 antibodies, but this negates the anti-tumor effect of the PDT/epacadostat combination, making its efficacy comparable to PDT alone (18). While this side effect is concerning, other studies have inhibited IDO through other methods without exhibiting the same toxicity. In one study, IDO was inhibited with a protoporphyrin IX and NLG919 conjugate in mouse models inoculated with breast cancer cells (47). This amplified PDT's immune response in tumor cells without significant toxicity of major organs (47). This study did not measure changes in Treg levels, but did report increased CT8+ T lymphocyte levels (47), implying that decreased Tregs likely played a role in augmented anti-tumor immunity. The role that IDO plays in the regulation of inflammation, both within the tumor and systemically, is poorly understood, and this method requires further investigation in preclinical and clinical trials to prevent toxic systemic side effects.

\section{Inhibition of MDSCs}

MDSCs are a heterogeneous population of immature myeloid cells which have been implicated to play important roles not only in pathological conditions, including cancer progression, but also in impacting the efficacy of anti-cancer agents (48-50). Importantly, these MDSC-induced effects are largely governed by their ability to induce immunosuppression, mediated via the orchestration of multiple signaling pathways as well as interactions with several immune cells and mediators (51-53). Therefore, strategies to target MDSCs have been hypothesized as one of the promising approaches to overcome immunosuppressive effects, restore anti-tumoral immunity response and/or enhance the efficacy of therapeutic agents. However, this is closely tied to the depletion of other immunophenotypes such as Tregs, thus, it is difficult, if not impossible, to affect one without affecting the other cell type(s).

One study by Korbelik et al revealed an improved cure rate in squamous cell carcinomas when all trans retinoic acid (ATRA) was administered with a vaccine made from tumor cells treated with PDT (17). In this study, squamous cell carcinoma cells were treated with PDT and injected into mice bearing the same squamous cell carcinoma tumors. Mice were also injected with ATRA, whose purpose was to facilitate the conversion of immunosuppressive MDSCs to a non-suppressive phenotype (54). In this study, ATRA reduced the number of MDSCs by causing their differentiation into mature myeloid cells, and overall made the PDT vaccine more effective by extending the time that PDT slowed the growth of the tumor (17). Thus, decreasing the MDSC population allows PDT to be effective against tumor cells for a longer period of time and reduce its overall size.

Reversing a hypoxic state in a tumor was also shown to impede the MDSC-regulated pathway $(55,56)$. Hypoxia is produced by consuming oxygen after making ATP (adenosine triphosphate), thus, reversing it can be achieved by interfering with oxidative phosphorylation $(57,58)$. This study used platelet membranes as nano-carriers called PM-IR780-Met, which included encapsulated metformin whose role was to decrease oxygen consumption by inhibiting the mitochondrial respiratory chain. This ultimately reduced the levels of MDSCs and their infiltration into tumor tissues. In turn, this reduced the number of Tregs being recruited by MDSCs and increased the infiltration of effector $\mathrm{T}$ cells into the tumor, lymph nodes, and spleen (56). Thus, it can be inferred that the nano-carrier increased both the anti-tumor and systemic immune responses. Furthermore, treatment with PDT without the nanocarrier decreased tumor growth from 7.5-fold to 4-fold, but the addition of metformin caused growth to decrease to 1.1-fold, showing a superior anti-tumor response (56). In addition to preventing the immunosuppressive effects of PDT, a constant supply of oxygen was supplied by the nano-carrier, and this allowed more ROS to be generated during PDT, rendering PDT more effective against tumors (56).

Sorafenib administered with low-dose PDT has been exploited as another method that enhances the T cell-mediated antitumor effects. Sorafenib has been shown to reduce MDSC and Treg populations $(28,59)$, while recruiting more antigen-processing cells and cytotoxic $\mathrm{T}$ cells to the tumor (28). When combined with PDT, sorafenib increases T-cell infiltration and inhibits tumor growth more effectively than PDT alone, and in some cases decreases tumor size (28). This was attributed to Sorafenib's ability to limit the interaction between cytotoxic CD8+ T cells and immunosuppressive cells, inducing a stronger anti-tumor immune response.

\section{Inhibition of COX-2}

As discussed earlier, PDT has been shown in mice to generate systemic immunosuppression through the lipid mediator PAF in a pathway leading to increased COX-2 expression and levels of Tregs $(33,60)$. However, COX-1, which is constitutively expressed in most cells, is not increased by PDT (60). Eicosanoids and COX-2-generated prostaglandins such as PGE2 have also been linked to local immunosuppression $(61,62)$. This pathway has not yet been demonstrated in humans. Given the availability of COX inhibitors, this strategy could serve as an easy target to combat the PDT-induced immunosuppression and relapse of pre-cancerous cells. In particular, selective COX-2 inhibitors such as celecoxib are safe for short-term use and may also decrease the painful side effects of PDT, so this method merits additional investigation.

Although not yet tested in humans, combination therapies involving selective COX-2 inhibitors have been shown to improve the therapeutic effectiveness of PDT in treating solid tumors in mice $(35,39,60,63)$. In one example, NS-398, a COX-2 inhibitor, was given in combination with PDT, and caused decreased levels of PGE2 and VEGF, which enhanced PDT's response in tumor cells of mouse carcinomas and 
sarcomas and resulted in a significant increase in tumor cures (compared to PDT alone) (60). Furthermore, the combination did not cause an increased response in non-tumor cells; specifically, it did not affect skin sensitization nor did it cause increased skin damage in sites without tumor cells (60).

In a second example by Makowski et al, there was no increased PDT efficacy in vitro when tumor cells were incubated with COX-2 inhibitors (39). This result was unexpected, especially following the results of Ferrario et al that showed PDT's effect potentiated by the addition of a COX-2 inhibitor (60). This prompted the group to conduct two different in vivo experiments: For one set of mice the COX-2 inhibitor before PDT, and the other received it chronically after illumination with PDT. The former showed no increased anti-tumor response, but with the latter, there was a statistically significant retardation of a poorly differentiated colon adenocarcinoma C-26 tumor growth, increased mouse survival, and higher complete cure rate compared to PDT alone (39). The proposed mechanism by Makowski et al is that the COX-2 inhibitor decreases angiogenic factors-which is synergistic with PDT's ability to cause vascular damage-and triggers apoptosis in tumor cells (39). This would explain why the anti-tumor effects were only increased when COX-2 was administered after PDT, since the tumor would have more difficulty repairing blood vessels following the vascular damage caused by PDT. This study demonstrated that COX-2 inhibitors may improve the efficacy of PDT through methods other than inhibiting immunosuppression.

Diclofenac is a nonsteroidal anti-inflammatory drug (NSAID) that functions as a cyclooxygenase-1 (COX-1) and COX-2 inhibitor. It has been shown in small clinical trials to improve the efficacy of PDT in reducing actinic keratoses when used as an adjuvant therapy, likely by targeting COX-2 receptors on actinic keratosis (64). Although not a COX-2 selective inhibitor, diclofenac shows promise because it is already used as a treatment on its own for actinic keratosis (33). Adjuvant therapy would not only make the treatment more effective, but also inhibit the immunosuppression caused by PDT. However, more patients reported pain, sometimes unbearable, and side effects such as pruritus, scaling, and crusting during PDT when used in conjunction with diclofenac (64). This might result in the diclofenac and PDT combination being reserved for small areas of skin.

\section{Conclusions}

With a better understanding of the mechanisms of immunosuppression of PDT with ALA, we can inhibit them and offer patients more effective treatments with potentially fewer side effects. This is the first review to specifically address methods of inhibiting immunosuppression for PDT with ALA. However, multiple options may not be considered practical due to the risk of side effects. For example, ATRA is only regularly used by oncologists and cyclophosphamide is only commonly used by rheumatologists, nephrologists, and dermatologists. Although diclofenac might not be a popular option due to side effects, the fact that it potentiated the effects of PDT merits exploration of other non-selective COX-inhibitors that could be used in conjunction with PDT. The approaches that use anti-CD25, sorafenib, abatacept, and
COX-2 inhibitors are more realistic in most settings. There are likely other options, yet to be determined, that involve different pathways for blocking PDT-induced immunosuppression.

It should be noted that other promising strategies are being developed to augment tumor-specific production of PpIX to include the use of topical vitamin D analogues (65). One effect of vitamin D receptor activation involves its ability to increase Tregs $(66,67)$, which could result in an 'immunosuppressive phenotype'. However, combinations of topical vitamin D agonists (calcipotriol) with 5-FU chemotherapy appear to result in a more pro-inflammatory effect which has been reported to result in long-term remissions (68). Hence, the exact effects of vitamin $\mathrm{D}$ as an adjunct to PDT on the skin immune system is an area of future investigation. Additionally, topical and oral nicotinamide (vitamin B3) replenish cellular ATP after irradiation with UV light (69). Through this mechanism, it has been shown to reduce the immunosuppression associated with both high and low-dose PDT, making it more effective against actinic keratoses and nonmelanoma skin cancers (70-73). This is a useful discovery, as nicotinamide is low-cost, readily available, and has few, if any side effects (74). Finally, immunosuppression has been shown to decrease by simply reducing the rate of irradiation, perhaps because of the decreased oxygen consumption at lower rates $(75,76)$. In pre-clinical trials, lower rates of PDT $\left(15\right.$ or $\left.45 \mathrm{mWcm}^{-2}\right)$ were as effective as high-rate PDT $\left(75 \mathrm{mWcm}^{-2}\right)$ in clearing tumors (77).

Though the immunosuppressive pathways appear complex, there is considerable rationale for pharmacologic strategies to target this unwanted effect. Further investigations documenting the exact level and mechanisms of PDT-induced immunosuppression demonstrated in mice need to be pursued in humans. In addition, there is a need for studies testing both the ability of various strategies such as COX inhibitors to inhibit PDT-induced immunosuppression with a clinical benefit such as improved clearance of actinic keratosis.

\section{Acknowledgements}

Not applicable.

\section{Funding}

This research was supported in part by grants from the National Institutes of Health (grant nos. R01 HL062996 and ES031087) and Veteran's Administration Merit Award (grant no. 5I01BX000853).

\section{Availability of data and materials}

Not applicable.

\section{Authors' contributions}

SB and CAR developed the concept and contributed to the majority of the manuscript writing. RPS and JBT made final revisions to the manuscript. Data authentication is not applicable. All authors have read and approved the final manuscript. 


\section{Ethics approval and consent to participate}

Not applicable.

\section{Patient consent for publication}

Not applicable.

\section{Competing interests}

The authors declare that they have no competing interests.

\section{References}

1. Dougherty TJ and Marcus SL: Photodynamic therapy. Eur Cancer 28: 1734-1742, 1992.

2. Sharman WM, Allen CM and van Lier JE: Photodynamic therapeutics: Basic principles and clinical applications. Drug Disc Today 4: 507-517, 1999.

3. Ochsner M: Photophysical and photobiological processes in the photodynamic therapy of tumours. J Photochem Photobiol B 39: $1-18,1997$.

4. Moan J: Porphyrin photosensitization and phototherapy. Photochem Photobiol 43: 681-690, 1986.

5. Allison RR, Downie GH, Cuenca R, Hu XH, Childs CJ and Sibata CH: Photosensitizers in clinical PDT. Photodiagnosis Photodyn Ther 1: 27-42, 2004.

6. Kwiatkowski S, Knap B, Przystupski D, Saczko J, Kędzierska E Knap-Czop K, Kotlińska J, Michel O, Kotowski K and Kulbacka J: Photodynamic therapy-mechanisms, photosensitizers and combinations. Biomed Pharmacother 106: 1098-1107, 2018.

7. Abrahamse $\mathrm{H}$ and Hamblin MR: New photosensitizers for photodynamic therapy. Biochem J 473: 347-364, 2016.

8. Zhang J, Jiang C, Figueiró Longo JP, Azevedo RB, Zhang H and Muehlmann LA: An updated overview on the development of new photosensitizers for anticancer photodynamic therapy. Acta Pharm Sin B 8: 137-146, 2018.

9. Chatterjee DK, Fong LS and Zhang Y: Nanoparticles in photodynamic therapy: An emerging paradigm. Adv Drug Deliv Rev 60 : $1627-1637,2008$

10. Gold M: ALA-PDT and MAL-PDT: What makes them different J Clin Aesthet Dermatol 2: 44-47, 2009.

11. Cantisani C, Paolino G, Faina V, Frascani F, Cantoresi F, Bianchini D, Gilda Fazia and Stefano Calvieri: Overview on topical 5-ALA photodynamic therapy use for non melanoma skin cancers. Int J Photoen 2014: 304862, 2014.

12. Morton CA and Braathen LR: Daylight photodynamic therapy for actinic keratoses. Am J Clin Dermatol 19: 647-656, 2018

13. Wehner MR: Comparing the efficacy of field treatments for actinic keratosis: A critical appraisal of a randomized trial in the New England Journal of Medicine. Br J Dermatol 182: 1343-1344, 2020.

14. Taub AF: Photodynamic therapy in dermatology: History and horizons. J Drugs Dermatol 3 (Suppl 1): S8-S25, 2004.

15. Armbrecht AM: A prospective study of visual function and quality of life following PDT in patients with wet age related macular degeneration. Br J Ophthalmol 88: 1270-1273, 2004.

16. McBride G: Studies expand potential uses of photodynamic therapy. J Natl Cancer Inst 94: 1740-1742, 2002

17. Korbelik M, Banáth J and Saw K: Immunoregulatory cell depletion improves the efficacy of photodynamic therapy-generated cancer vaccines. Int J Mol Sci 16: 27005-27014, 2015.

18. Wachowska M, Stachura J, Tonecka K, Fidyt K, Braniewska A, Sas Z, Kotula I, Rygiel TP, Boon L, Golab J and Muchowicz A Inhibition of IDO leads to IL-6-dependent systemic inflammation in mice when combined with photodynamic therapy. Cancer Immunol Immunother 69: 1101-1112, 2020.

19. Reddan JC, Anderson CY, Xu H, Hrabovsky S, Freye K, Fairchild R, Tubesing KA and Elmets CA: Immunosuppressive effects of silicon phthalocyanine photodynamic therapy. Photochem Photobiol 70: 72-77, 1999.

20. Lynch DH, Haddad S, King VJ, Ott MK, Straight R and Jolles CJ: Systemic immunosuppression induced by photodynamic therapy (PDT) is adoptively transferred by macrophages. Photochem Photobiol 49: 453-458, 1989.
21. Reginato E, Mroz P, Chung H, Kawakubo M, Wolf $\mathrm{P}$ and Hamblin MR: Photodynamic therapy plus regulatory T-cell depletion produces immunity against a mouse tumour that expresses a self-antigen. Br J Cancer 109: 2167-2174, 2013.

22. Mroz P and Hamblin MR: The immunosuppressive side of PDT Photochem Photobiol Sci 10: 751-758, 2011.

23. Falk-Mahapatra R and Gollnick SO: Photodynamic therapy and immunity: An update. Photochem Photobiol 96: 550-559, 2020.

24. Nobbe S, Trüeb RM, French LE and Hofbauer GFL: Herpes simplex virus reactivation as a complication of photodynamic therapy. Photodermatol Photoimmunol Photomed 27: 51-52, 2011.

25. Wolf P, Fink-Puches R, Reimann-Weber A and Kerl H: Development of malignant melanoma after repeated topical photodynamic therapy with 5-Aminolevulinic acid at the exposed site. Dermatology 194: 53-54, 1997.

26. Fiechter S, Skaria A, Nievergelt H, Anex R, Borradori L and Parmentier L: Facial basal cell carcinomas recurring after photodynamic therapy: A retrospective analysis of histological subtypes. Dermatology 224: 346-351, 2012.

27. Castano AP, Mroz P, Wu MX and Hamblin MR: Photodynamic therapy plus low-dose cyclophosphamide generates antitumor immunity in a mouse model. Proc Natl Acad Sci USA 105 5495-5500, 2008.

28. Sun X, Cao Z, Mao K, Wu C, Chen H, Wang J, Wang X, Cong X, Li Y, Meng X, et al: Photodynamic therapy produces enhanced efficacy of antitumor immunotherapy by simultaneously inducing intratumoral release of sorafenib. Biomaterials 240: 119845,2020

29. Lindau D, Gielen P, Kroesen M, Wesseling P and Adema GJ: The immunosuppressive tumour network: Myeloid-derived suppressor cells, regulatory $\mathrm{T}$ cells and natural killer $\mathrm{T}$ cells. Immunology 138: 105-115, 2013.

30. Wang Y, Ma Y, Fang Y, Wu S, Liu L, Fu D and Shen X: Regulatory T cell: A protection for tumour cells. J Cell Mol Med 16: 425-436, 2012.

31. Lee CR, Kwak Y, Yang T, Han JH, Park SH, Ye MB, Lee W, Sim KY, Kang JA, Kim YC, et al: Myeloid-derived suppressor cells are controlled by regulatory T cells via TGF- $\beta$ during murine colitis. Cell Rep 17: 3219-3232, 2016.

32. Fujimura T, Kambayashi Y and Aiba S: Crosstalk between regulatory $\mathrm{T}$ cells (Tregs) and myeloid derived suppressor cells (MDSCs) during melanoma growth. Onco Immunol 1: 1433-1434, 2012.

33. Ferracini M, Sahu RP, Harrison KA, Waeiss RA, Murphy RC, Jancar S, Konger RL and Travers JB: Topical photodynamic therapy induces systemic immunosuppression via generation of platelet-activating factor receptor ligands. J Invest Dermatol 135: 321-323, 2015.

34. Sahu RP, Petrache I, van Demark MJ, Rashid BM, Ocana JA, Tang Y, Yi Q, Turner MJ, Konger RL and Travers JB: Cigarette smoke exposure inhibits contact hypersensitivity via the generation of platelet-activating factor agonists. J Immunol 190: 2447-2454, 2013

35. Zhang Q, Yao Y, Konger RL, Sinn AL, Cai S, Pollok KE and Travers JB: UVB radiation-mediated inhibition of contact hypersensitivity reactions is dependent on the platelet-activating factor system. J Invest Dermatol 128: 1780-1787, 2008.

36. Zelenay S, van der Veen AG, Böttcher JP, Snelgrove KJ, Rogers N, Acton SE, Chakravarty P, Girotti MR, Marais R, Quezada SA, et al: Cyclooxygenase-dependent tumor growth through evasion of immunity. Cell 162: 1257-1270, 2015.

37. Ke J, Yang Y, Che Q, Jiang F, Wang H, Chen Z, Zhu M, Tong H, Zhang H, Yan X, et al: Prostaglandin E2 (PGE2) promotes proliferation and invasion by enhancing SUMO-1 activity via EP4 receptor in endometrial cancer. Tumor Biol 37: 12203-12211, 2016.

38. Fujita M, Kohanbash G, Fellows-Mayle W, Hamilton RL, Komohara Y, Decker SA, Ohlfest JR and Okada H: COX-2 blockade suppresses gliomagenesis by inhibiting myeloid-derived suppressor cells. Cancer Res 71: 2664-2674, 2011.

39. Makowski M, Grzela T, Niderla J, Łazarczyk M, Mróz P, Kopeé M, Legat M, Strusińska K, Koziak K, Nowis D, et al: Inhibition of cyclooxygenase-2 indirectly potentiates antitumor effects of photodynamic therapy in mice. Clin Cancer Res 9: 5417-5422, 2003

40. Togashi Y and Nishikawa H: Regulatory T cells: Molecular and cellular basis for immunoregulation. Curr Top Microbiol Immunol 410: 3-27, 2017. 
41. Najafi M, Farhood B and Mortezaee K: Contribution of regulatory T cells to cancer: A review. J Cell Physiol 234: 7983-7993, 2019.

42. Moreno Ayala MA, Li Z and DuPage M: Treg programming and therapeutic reprogramming in cancer. Immunology 157: 198-209, 2019.

43. Yan S, Zhang Y and Sun B: The function and potential drug targets of tumour-associated Tregs for cancer immunotherapy. Sci China Life Sci 62: 179-186, 2019.

44. Oh DS, Kim H, Oh JE, Jung HE, Lee YS, Park JH and Lee HK Intratumoral depletion of regulatory T cells using CD25-targeted photodynamic therapy in a mouse melanoma model induces antitumoral immune responses. Oncotarget 8: 47440-47453, 2017.

45. Mellor AL, Lemos H and Huang L: Indoleamine 2,3-Dioxygenase and tolerance: Where are we now? Front Immunol 8: 1360, 2017

46. Baban B, Chandler PR, Sharma MD, Pihkala J, Koni PA, Munn DH and Mellor AL: IDO activates regulatory T cells and blocks their conversion into Th17-Like T cells. J Immuno 183: 2475-2483, 2009

47. Huang Z, Wei G, Zeng Z, Huang Y, Huang L, Shen Y, Sun X, $\mathrm{Xu} \mathrm{C}$ and Zhao C: Enhanced cancer therapy through synergetic photodynamic/immune checkpoint blockade mediated by a liposomal conjugate comprised of porphyrin and IDO inhibitor. Theranostics 9: 5542-5557, 2019.

48. Consonni FM, Porta C, Marino A, Pandolfo C, Mola S, Bleve A and Sica A: Myeloid-derived suppressor cells: Ductile targets in disease. Front Immunol 10: 949, 2019.

49. Pawelec G, Verschoor CP and Ostrand-Rosenberg S: Myeloid-derived suppressor cells: Not only in tumor immunity. Front Immunol 10: 1099, 2019.

50. Zuo H, Hou Y, Yu Y, Li Z, Liu H, Liu C, He J and Miao L: Circumventing myeloid-derived suppressor cell-mediated immunosuppression using an oxygen-generated and -economized nanoplatform. ACS ACS Appl Mater Interfaces 12: 55723-55736, 2020.

51. Groth C, Hu X, Weber R, Fleming V, Altevogt P, Utikal J and Umansky V: Immunosuppression mediated by myeloid-derived suppressor cells (MDSCs) during tumour progression. $\mathrm{Br}$ J Cancer 120: 16-25, 2019.

52. Ostrand-Rosenberg S, Sinha P, Beury DW and Clements VK: Cross-talk between myeloid-derived suppressor cells (MDSC) macrophages, and dendritic cells enhances tumor-induced immune suppression. Semin Cancer Biol 22: 275-281, 2012.

53. Bruno A, Mortara L, Baci D, Noonan DM and Albini A: Myeloid derived suppressor cells interactions with natural killer cells and pro-angiogenic activities: Roles in tumor progression. Fronti Immunol 10: 771, 2019.

54. Iclozan C, Antonia S, Chiappori A, Chen DT and Gabrilovich D Therapeutic regulation of myeloid-derived suppressor cells and immune response to cancer vaccine in patients with extensive stage small cell lung cancer. Cancer Immunol Immunother 62: 909-918, 2013

55. Larue L, Myrzakhmetov B, Ben-Mihoub A, Moussaron A, Thomas N, Arnoux P, Baros F, Vanderesse R, Acherar S and Frochot C: Fighting hypoxia to improve PDT. Pharmaceuticals (Basel) 12: 163, 2019.

56. Mai X, Zhang Y, Fan H, Song W, Chang Y, Chen B, Shi J, Xin $X$, Teng Z, Sun J and Teng G: Integration of immunogenic activation and immunosuppressive reversion using mitochondrial-respiration-inhibited platelet-mimicking nanoparticles. Biomaterials 232: 119699, 2020.

57. Zhou Z, Zhang B, Wang H, Yuan A, Hu Y and Wu J: Two-stage oxygen delivery for enhanced radiotherapy by perfluorocarbon nanoparticles. Theranostics 8: 4898-4911, 2018.

58. Ji C, Lu Z, Xu Y, Shen B, Yu S and Shi D: Self-production of oxygen system $\mathrm{CaO}_{2} / \mathrm{MnO}_{2} @$ PDA-MB for the photodynamic therapy research and switch-control tumor cell imaging. J Biomed Mater Res B Appl Biomater 106: 2544-2552, 2018.

59. Cao M, Xu Y, Youn J, Cabrera R, Zhang X, Gabrilovich D, Nelson DR and Liu C: Kinase inhibitor Sorafenib modulates immunosuppressive cell populations in a murine liver cancer model. Lab Invest 91: 598-608, 2011.

60. Ferrario A, von Tiehl K, Wong S, Luna M and Gomer CJ: Cyclooxygenase-2 inhibitor treatment enhances photodynamic therapy-mediated tumor response. Cancer Res 62: 3956-3961, 2002.
61. Zhang Z, Huang S, Wu S, Qi J, Li W, Liu S, Cong Y, Chen H, Lu L, Shi S, et al: Clearance of apoptotic cells by mesenchymal stem cells contributes to immunosuppression via PGE2. EBioMedicine 45: 341-350, 2019.

62. Kim SH, Roszik J, Cho S-N, Ogata D, Milton DR, Peng W, Menter DG, Ekmekcioglu S and Grimm EA: The COX2 effector microsomal PGE2 synthase 1 is a regulator of immunosuppression in cutaneous melanoma. Clin Cancer Res 25: 1650-1663, 2019.

63. Ferrario A and Gomer CJ: Targeting the tumor microenvironment using photodynamic therapy combined with inhibitors of cyclooxygenase- 2 or vascular endothelial growth factor. Methods Mol Biol 635: 121-132, 2010.

64. Van der Geer S and Krekels GAM: Treatment of actinic keratoses on the dorsum of the hands: ALA-PDT versus diclofenac 3\% gel followed by ALA-PDT. A placebo-controlled, double-blind, pilot study. J Dermatolog Treat 20: 259-265, 2009.

65. Rollakanti K, Anand S and Maytin EV: Topical calcitriol prior to photodynamic therapy enhances treatment efficacy in non-melanoma skin cancer mouse models. Proc SPIE Int Soc Opt Eng 9308: 93080Q, 2015.

66. Ni C, Gan X, Li X, Sun H, Chen Z and Lu H: Vitamin D alleviates acute graft-versus-host disease through promoting the generation of Foxp3+ T cells. Ann Transl Med 7: 748, 2019.

67. van der Aar AM, Sibiryak DS, Bakdash G, van Capel TM, van der Kleij HP, Opstelten DJ, Teunissen MB, Kapsenberg ML and de Jong EC: Vitamin D3 targets epidermal and dermal dendritic cells for induction of distinct regulatory $\mathrm{T}$ cells. J Allergy Clin Immunol 127: 1532-1540.e7, 2011.

68. Rosenberg AR, Tabacchi M, Ngo KH, Wallendorf M, Rosman IS Cornelius LA and Demehri S: Skin cancer precursor immunotherapy for squamous cell carcinoma prevention. JCI Insight 4: e125476, 2019.

69. Park J, Halliday GM, Surjana D and Damian DL: Nicotinamide prevents ultraviolet radiation-induced cellular energy loss. Photochem Photobiol 86: 942-948, 2010.

70. Gensler HL: Prevention of photoimmunosuppression and photocarcinogenesis by topical nicotinamide. Nutr Cancer 29: 157-162, 1997.

71. Gensler HL, Williams T, Huang AC and Jacobson EL: Oral niacin prevents photocarcinogenesis and photoimmunosuppression in mice. Nutr Cancer 34: 36-41, 1999.

72. Thanos SM, Halliday GM and Damian DL: Nicotinamide reduces photodynamic therapy-induced immunosuppression in humans. Br J Dermatol 167: 631-636, 2012.

73. Surjana D, Halliday GM, Martin AJ, Moloney FJ and Damian DL: Oral nicotinamide reduces actinic keratoses in phase II double-blinded randomized controlled trials. J Invest Dermatol 132: 1497-1500, 2012.

74. Knip M, Douek IF, Moore WPT, Gillmor HA, McLean AEM, Bingley PJ and Gale EA; European Nicotinamide Diabetes Intervention Trial Group: Safety of high-dose nicotinamide: A review. Diabetologia 43: 1337-1345, 2000.

75. Foster TH, Murant RS, Bryant RG, Knox RS, Gibson SL and Hilf R: Oxygen consumption and diffusion effects in photodynamic therapy. Radiat Res 126: 296-303, 1991.

76. Frost GA, Halliday GM and Damian DL: Photodynamic therapy-induced immunosuppression in humans is prevented by reducing the rate of light delivery. J Invest Dermatol 131: 962-968, 2011.

77. Ericson MB, Sandberg C, Stenquist B, Gudmundson F, Karlsson M, Ros A-M, Rosén A, Larkö O, Wennberg AM and Rosdahl I: Photodynamic therapy of actinic keratosis at varying fluence rates: Assessment of photobleaching, pain and primary clinical outcome. Br J Dermatol 151: 1204-1212, 2004.

This work is licensed under a Creative Commons Attribution-NonCommercial-NoDerivatives 4.0 International (CC BY-NC-ND 4.0) License. 